\title{
Hypophysitis as a complication of jpilimumab treatment
}

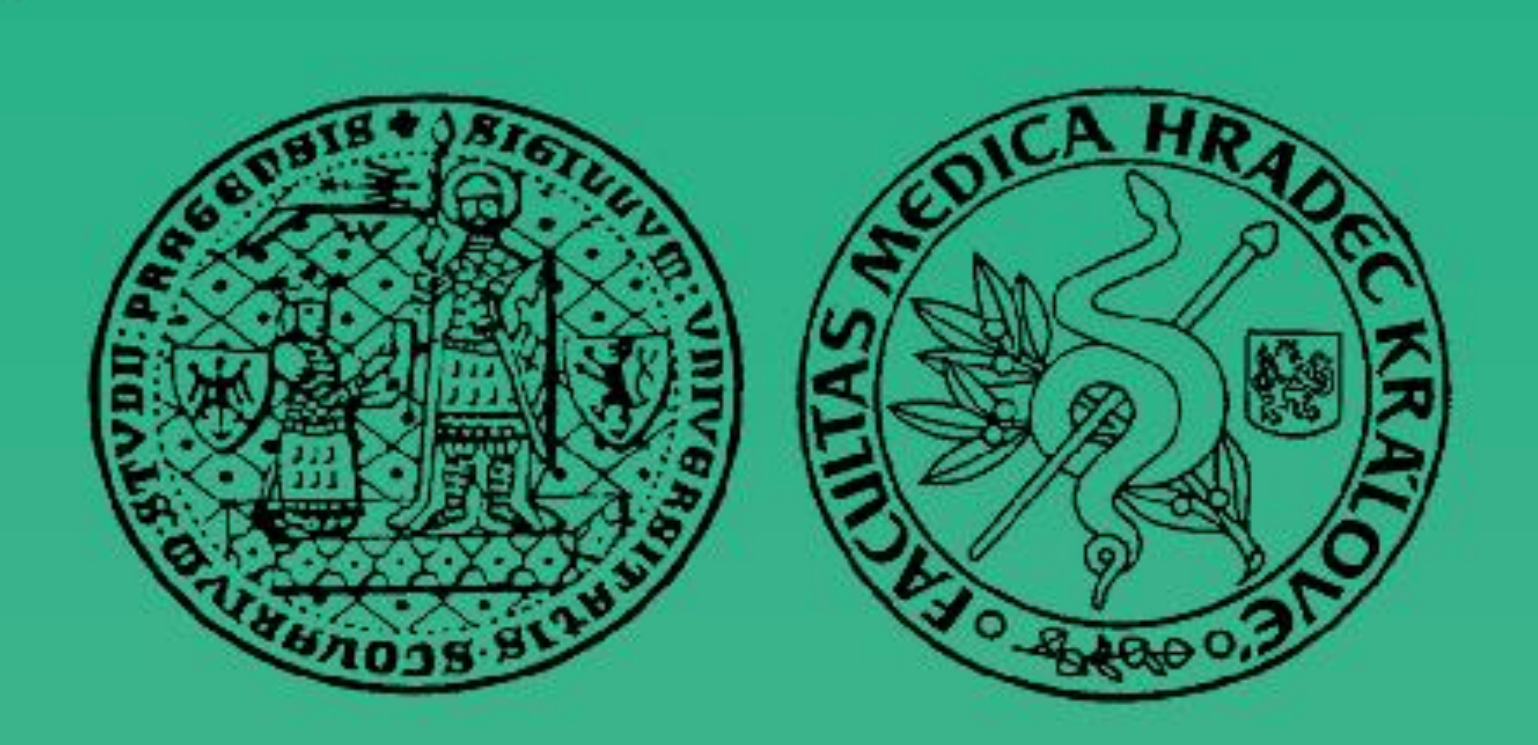

\author{
Svilias I. ${ }^{1}$, Čáp J.1 \\ 4th Dep. of Internal Medicine - Hematology and Endocrinology \\ Charles University Hospital and Faculty of Medicine in Hradec Králové
}

\section{Introduction}

In the last few years new immunomodulatory drugs are used for the treatment of metastatic melanoma. One of these drugs is ipilimumab, a monoclonal antibody that activates the immune system by targeting CTLA-4 protein receptor. This monoclonal antibody is very effective but there is a higher risk of endocrinopathies like an adverse effect of treatment, mostly hypophysitis and hypothyroidism.

Case

We present a 68 years old patient with metastatic melanoma after 4 cycles of ipilimumab treatment (picture 1)

She was admitted for malaise, headache, nausea and vomiting.

- Laboratory finding: cortisol lower than 28nmol/l, ACTH 12,7 ng/l (normal range 3,6-60,5ng/l), Na $128 \mathrm{mmol} / \mathrm{l}, \mathrm{Cl} 93 \mathrm{mmol} / \mathrm{l}$,

TSH 0,11 mU/l, Ft4 16,5 pmol/l (subclinical hyperthyroidism due to multinodular goitre, autoantibodies negative),

$\mathrm{PRL}, \mathrm{LH}, \mathrm{FSH}$ in the normal range

- Administration of corticosteroids i.v. was started with an immediate effect

- MR of hypophysis was performed 3 days after corticosteroid treatment with a normal result (picture 2 and 3 )

- Eleven months after hypophysitis the patient is still on corticosteroids

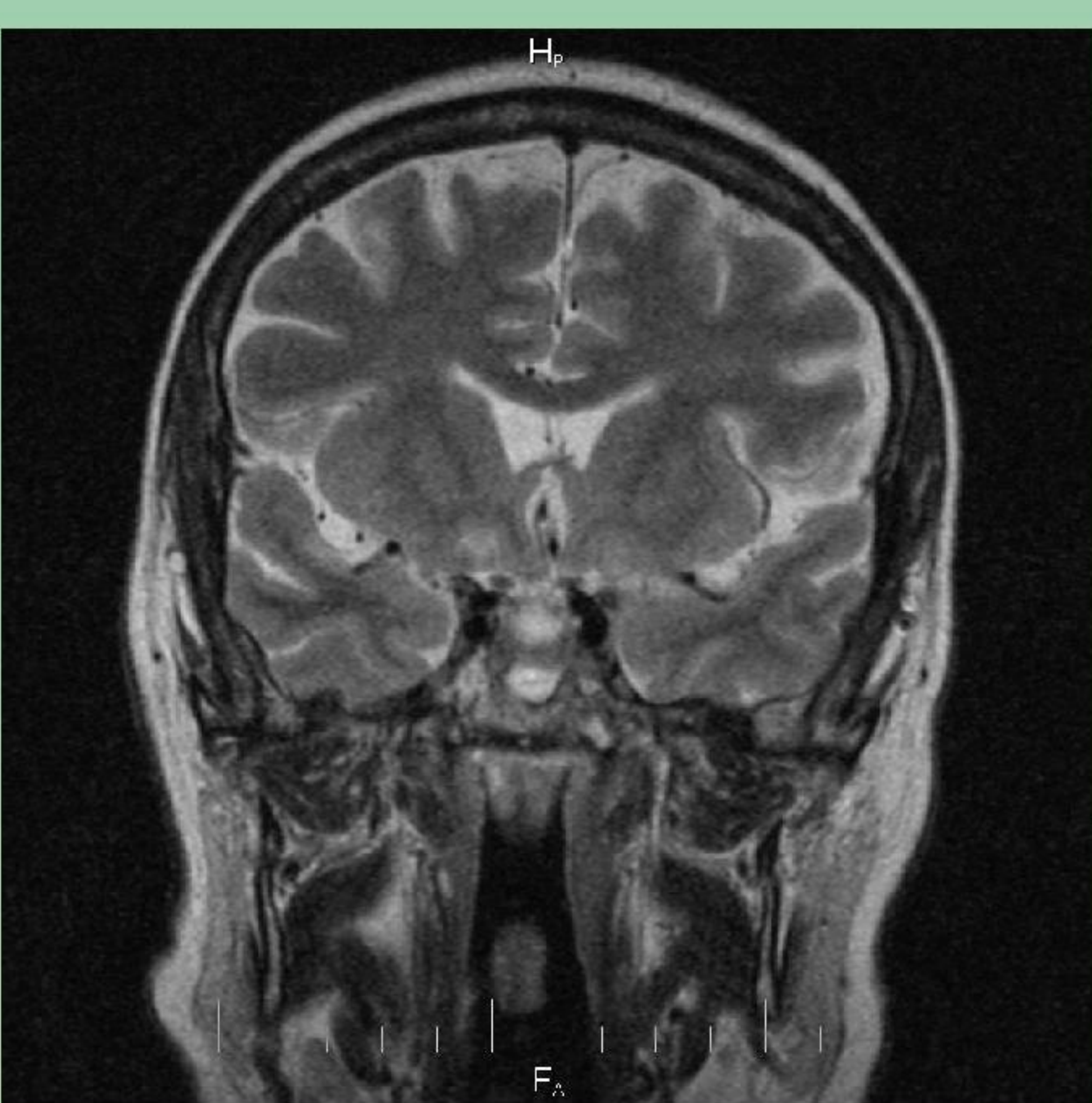

Picture 2. T2 cor MR hypophysis

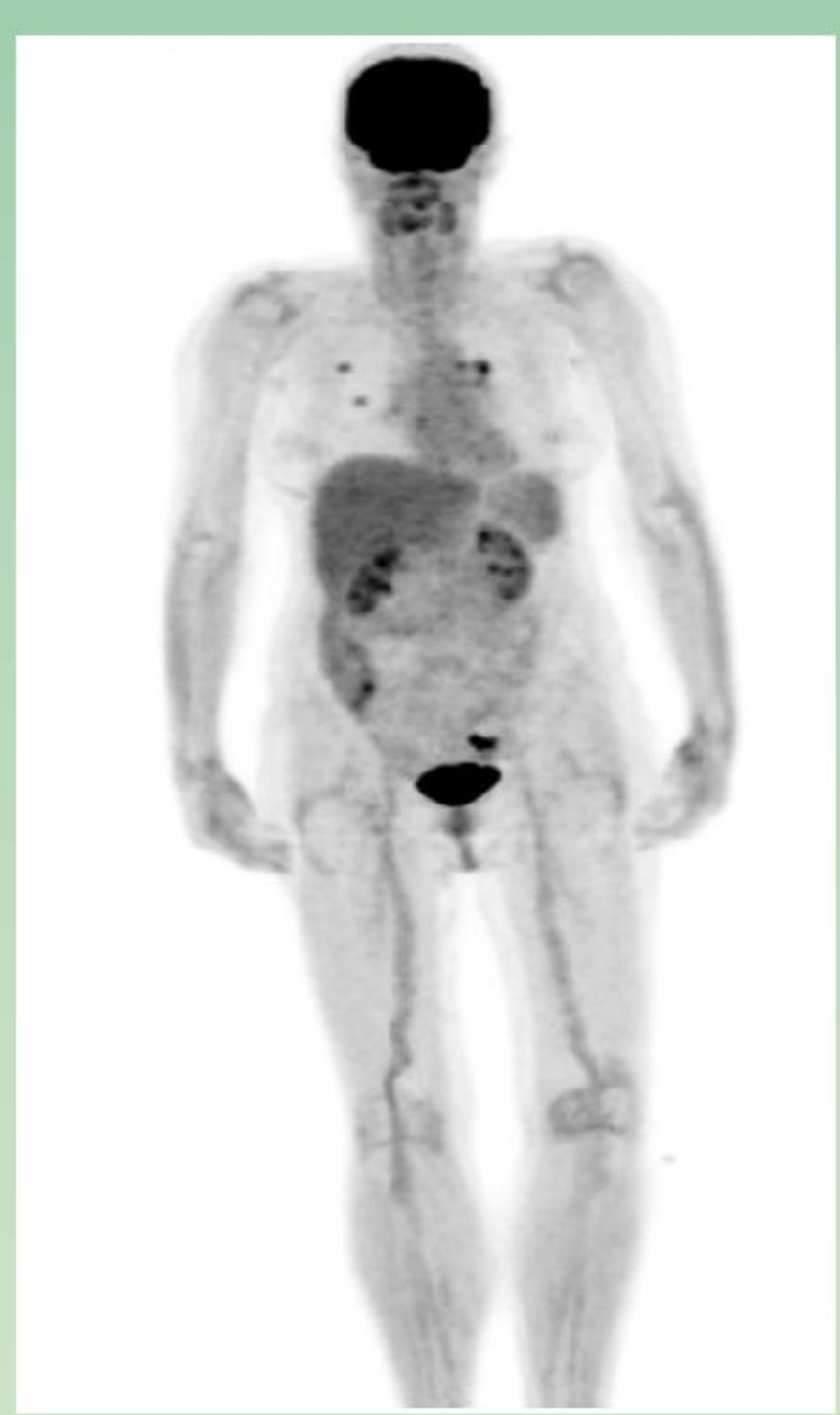

Picture $1 \mathrm{PET} / \mathrm{CT}$

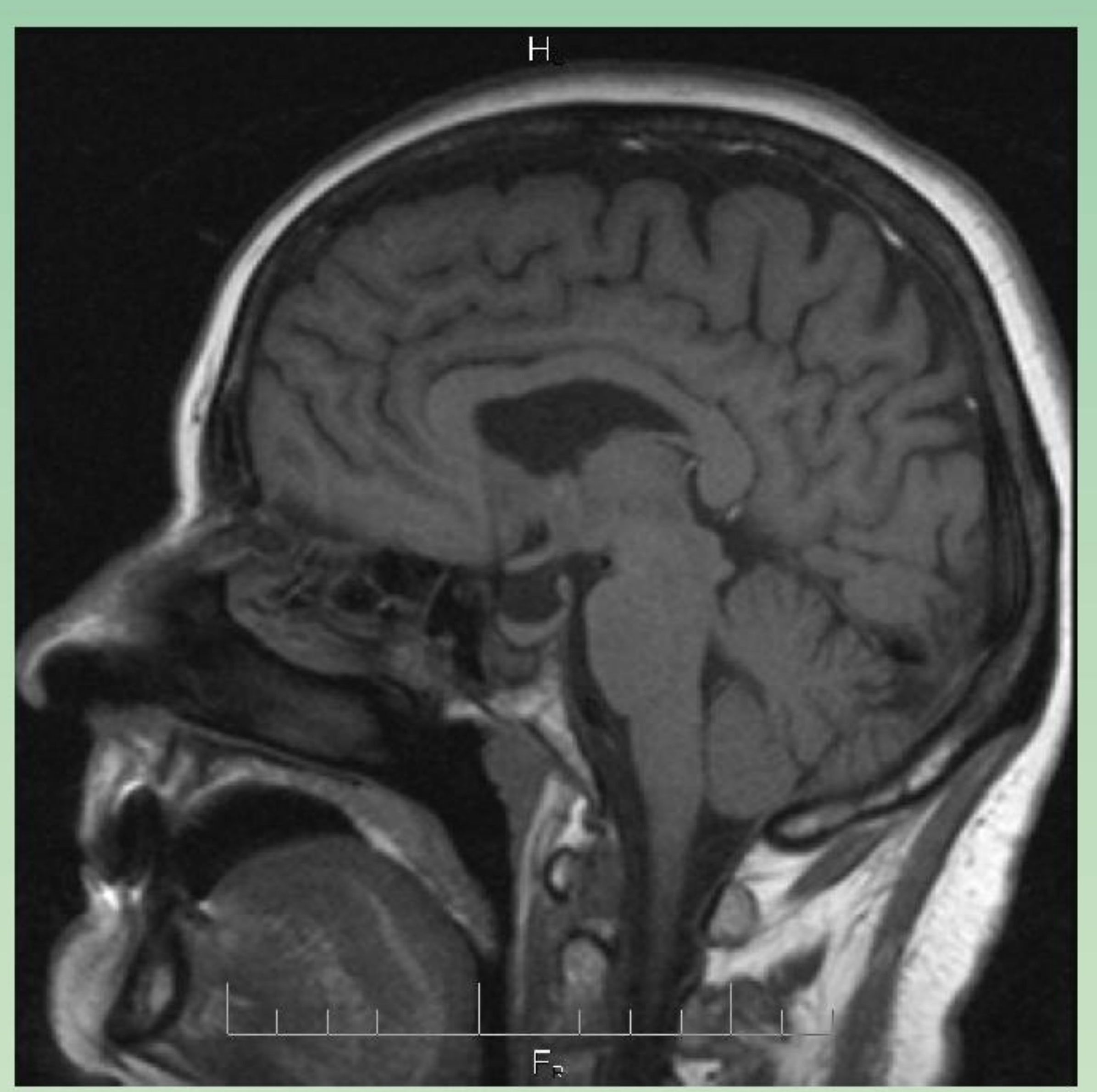

Picture 3 T1 sag MR hypophysis

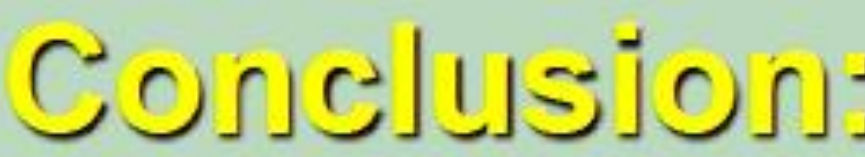

Ipilimumab is a very effective CTLA-4 inhibitor that prolongs survival in patients with metatstatic melanoma. In the literature cases of hypophysitis were described as an adverse effect of treatment. That's why during therapy they are necessary regular controls of pituitary hormones. In case of hypophysitis high-dose corticosteroids should be given. 